\title{
Predicting metastasis from cues of metastatic cancer stem-like cells-3D- ultrasensitive metasensor at a single-cell level
}

\author{
Authors \\ Priya Dharmalingam ${ }^{1,2,3,5}$, Krishnan Venkatakrishnan ${ }^{1,4}$, *, Bo Tan $^{3,4}$
}

\section{Affiliations}

${ }^{1}$ Ultrashort Laser Nano Manufacturing Research Facility, Department of Mechanical and Industrial Engineering, Ryerson University, 350 Victoria Street, Toronto, Ontario M5B 2K3,

Canada

${ }^{2}$ Institute for Biomedical Engineering, Science and Technology (I-BEST), Partnership between

Ryerson University and St.Michael’s Hospital, Toronto, Ontario M5B 1W8, Canada.

${ }^{3}$ Nano Characterization Laboratory, Department of Aerospace Engineering, Ryerson University, 350 Victoria Street, Toronto, Ontario M5B 2K3, Canada.

${ }^{4}$ Affiliate Scientist, Keenan Research Center, St. Michael’s Hospital, 209 Victoria Street, Toronto, Ontario, M5B 1T8, Canada

${ }^{5}$ Nano-Bio Interface Facility, Department of Mechanical and Industrial Engineering, Ryerson University, 350 Victoria Street, Toronto, Ontario M5B 2K3, Canada.

*Email: venkat@ryerson.ca 
a)

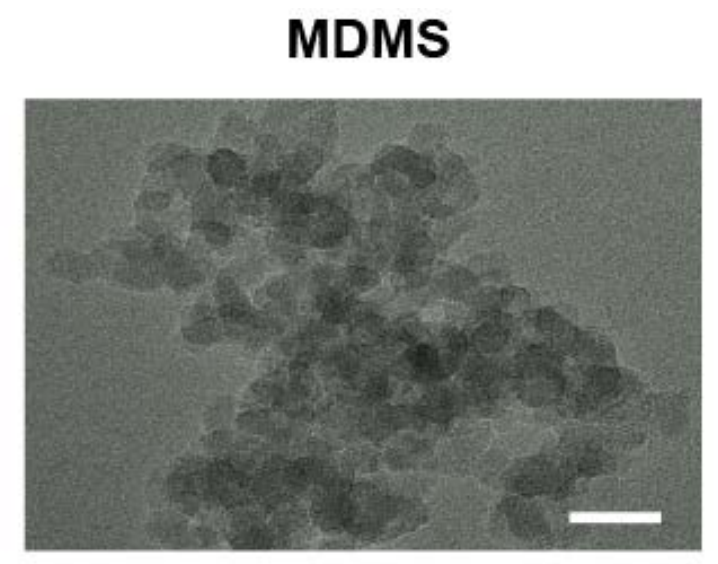

b)

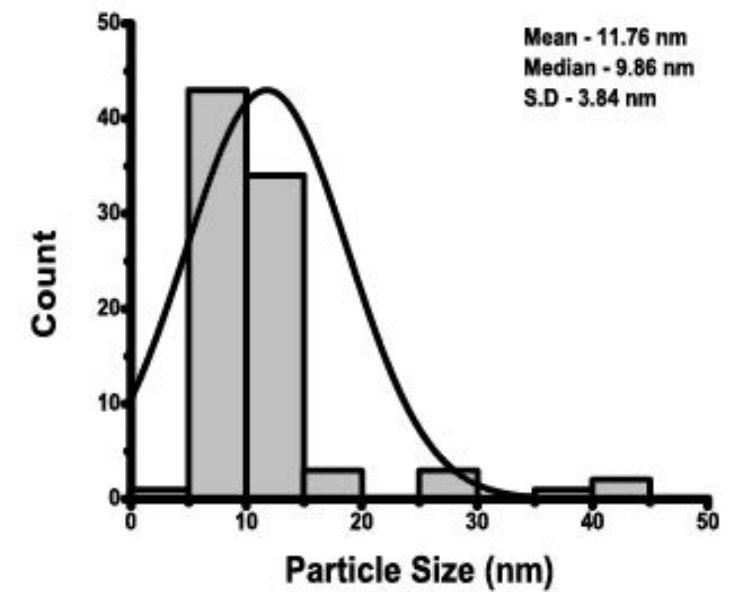

\section{PDMS}

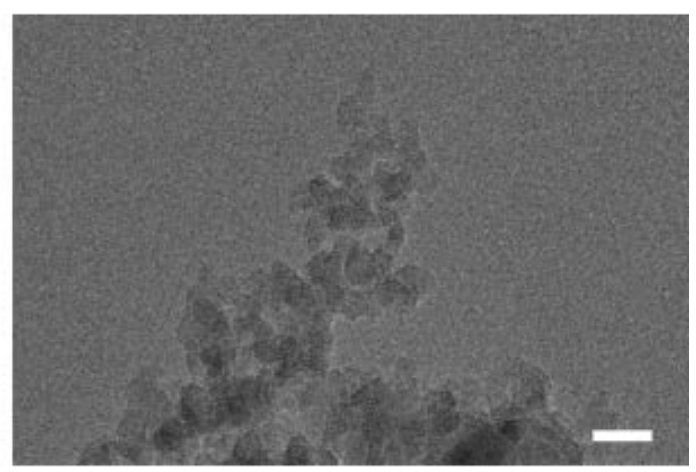

PDMS

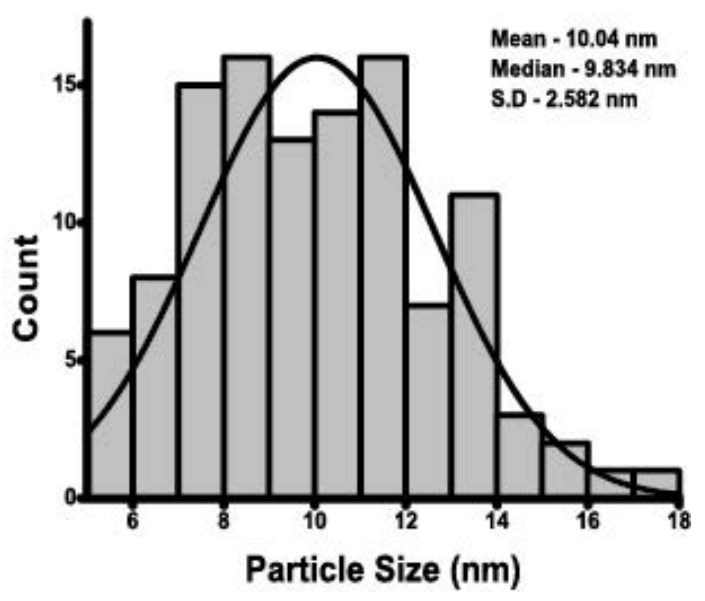

Figure S1: TEM and size distribution analysis of the MDMS and PDMS sensors. 


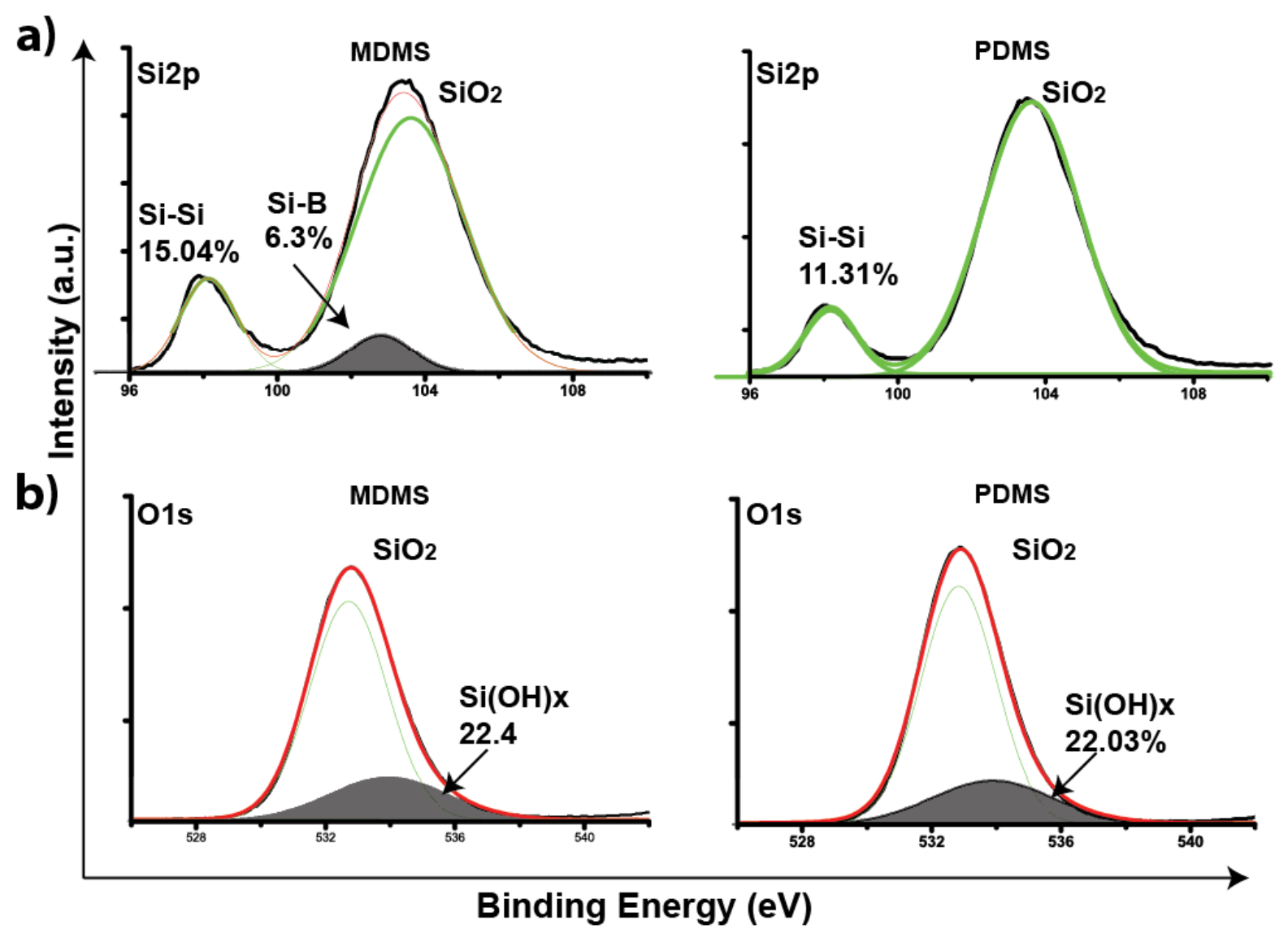

Figure S2: XPS analysis investigates Si2p and O1s of MDMS and PDMS.

a)

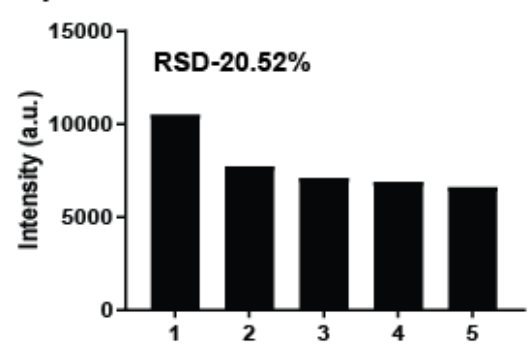

d)

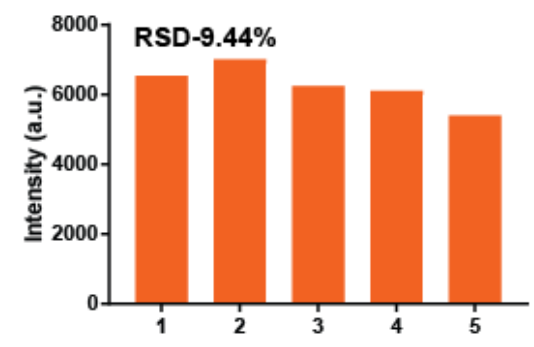

b) $\quad 776 \mathrm{~cm}^{-1}$

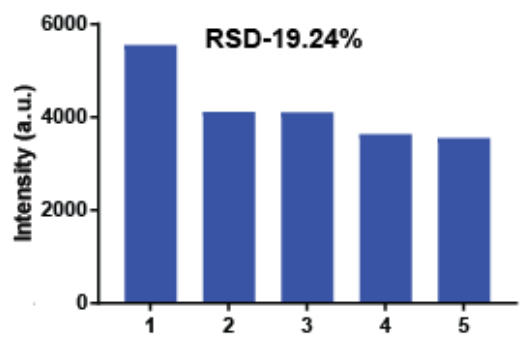

e)

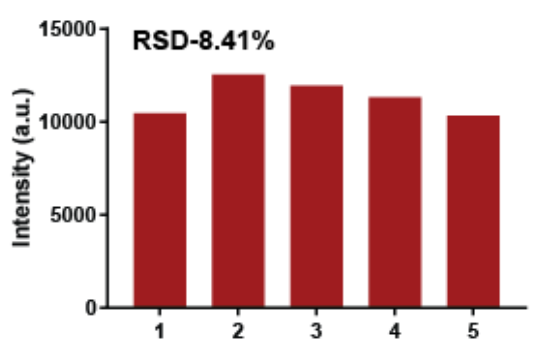

C) $1188 \mathrm{~cm}^{-1}$
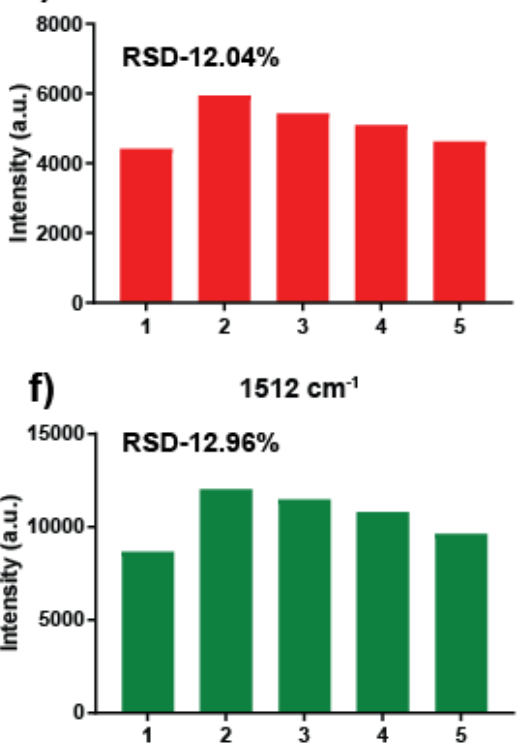

Figure S3: HDMS reproducibility at mM concentration of R6G analyte molecule. 
a)

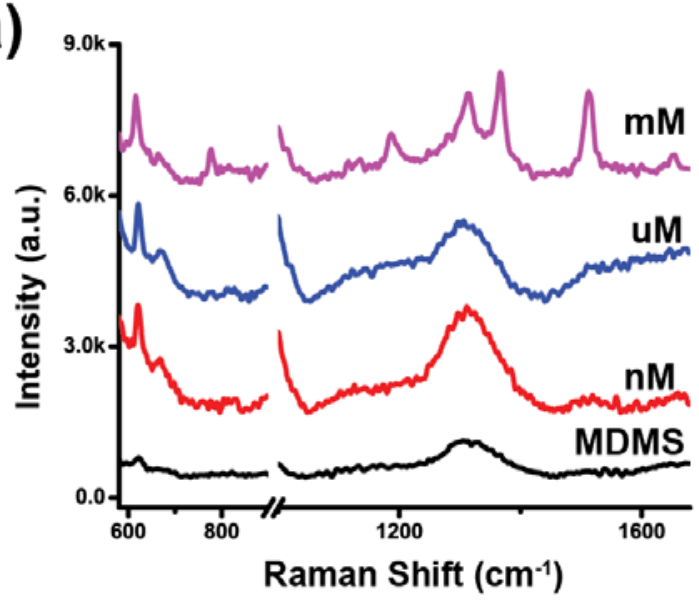

b)

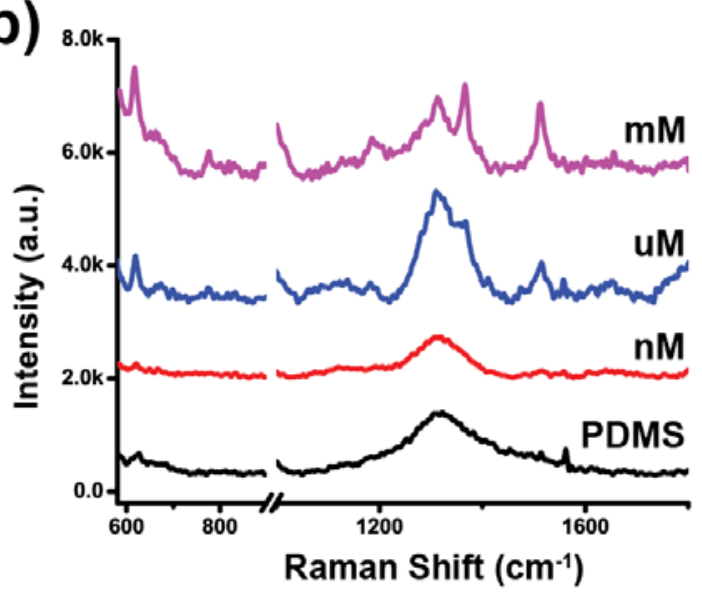

Figure S4: a-b) Detection efficiency of R6G molecule tested in MDMS and PDMS.

a)
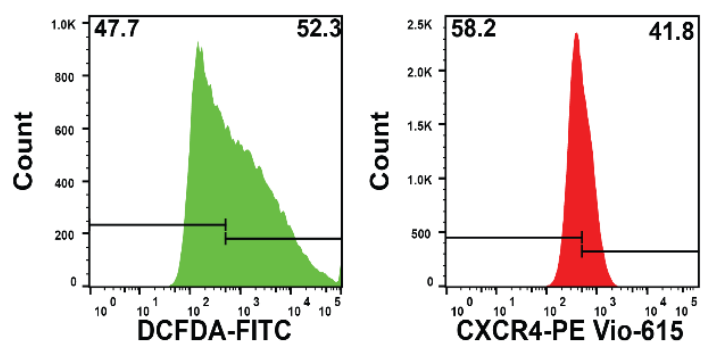

c)

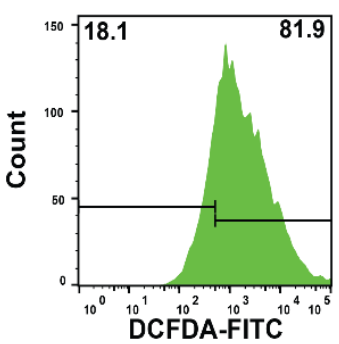

b)
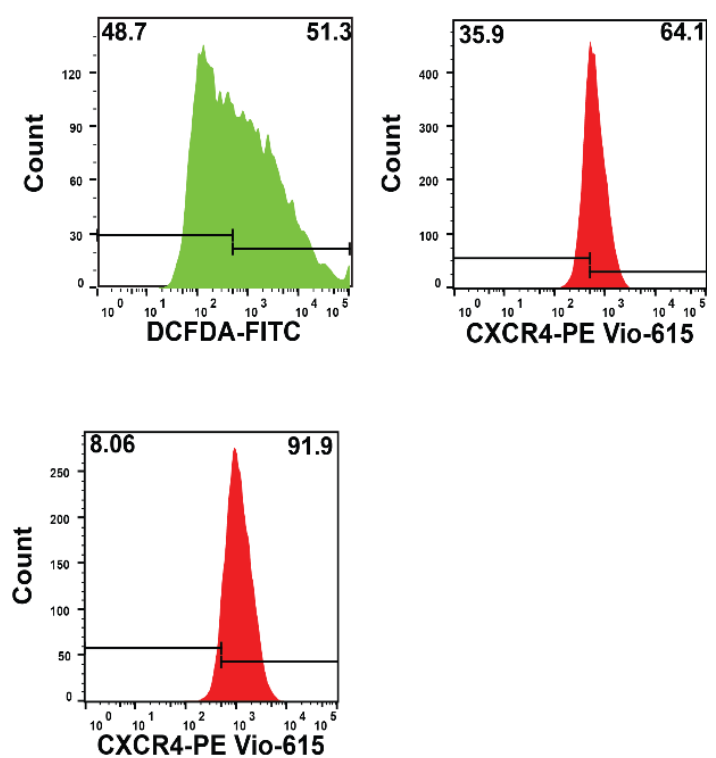

Figure S5: Histogram shows overall progenitors' population (CXCR4, ROS) studied from different levels of cancer types. 
a)

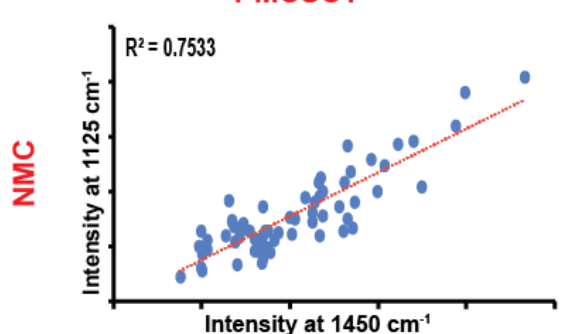

b)

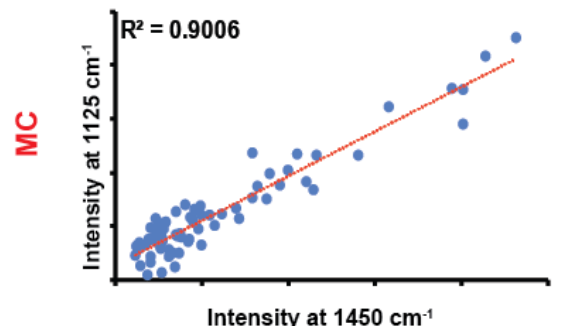

c)

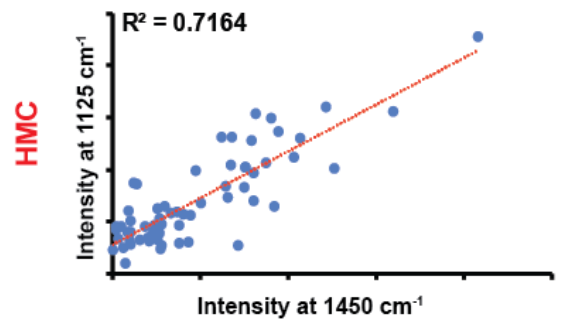

PMCSC2
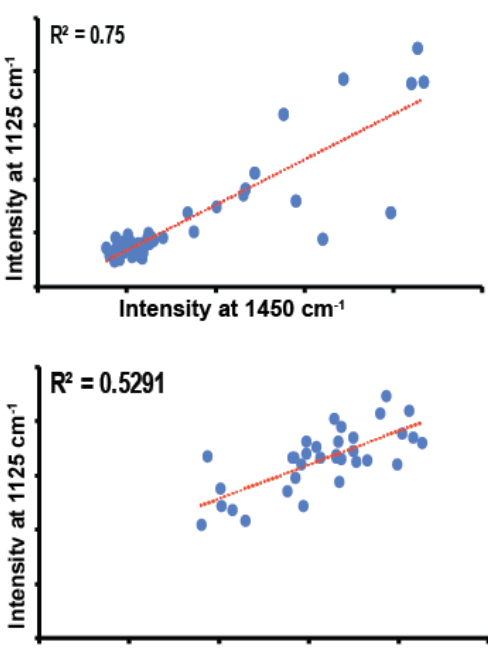

Intensity at $1450 \mathrm{~cm}^{-1}$

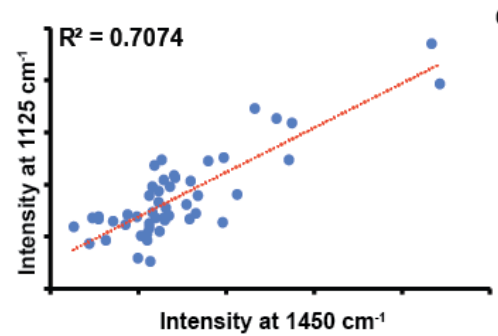

NMCSC
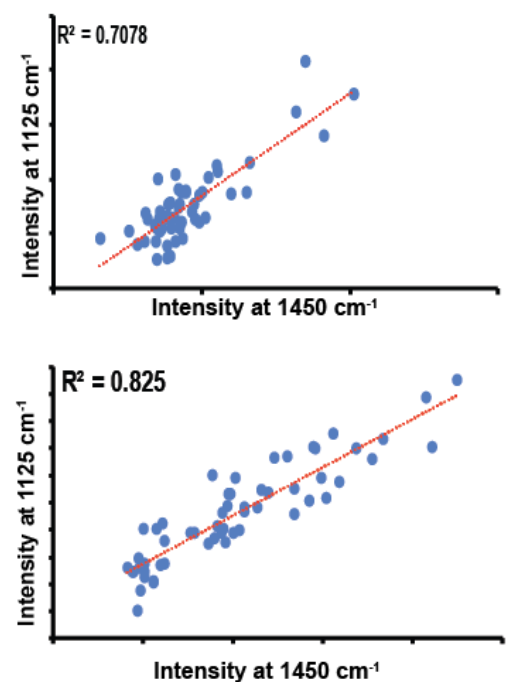

d)

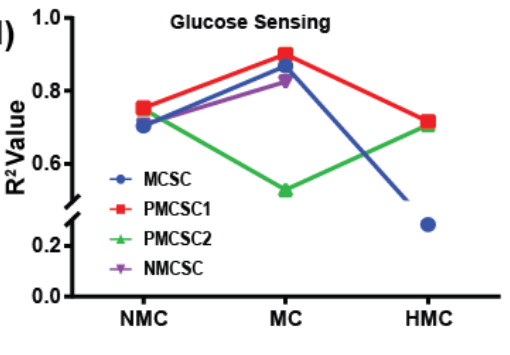

Figure S6: Single-cell regression analysis of glucose-metabolite signals from different cancers progeny and non-metastatic stem cell populations. 
a)

b)

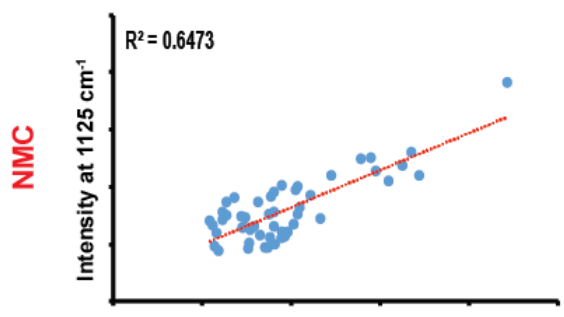

Intensity at $866 \mathrm{~cm}^{-1}$

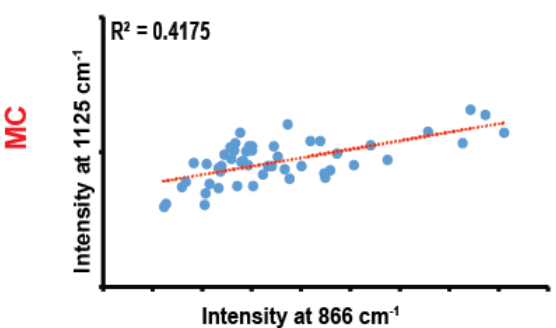

c)

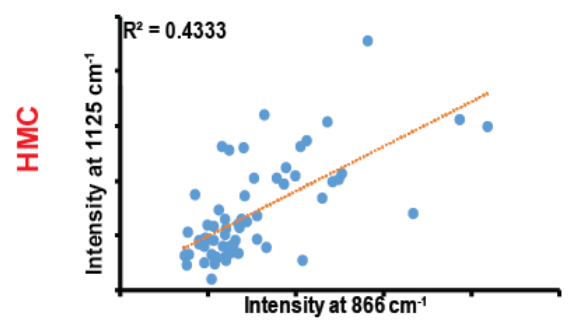

PMCSC2

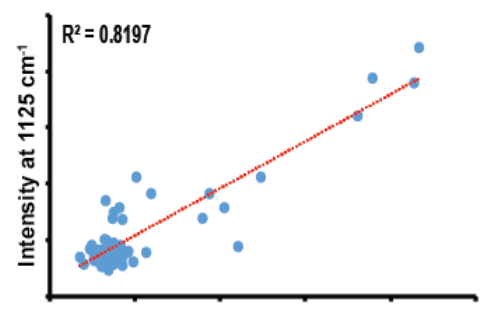

Intensity at $866 \mathrm{~cm}^{-1}$
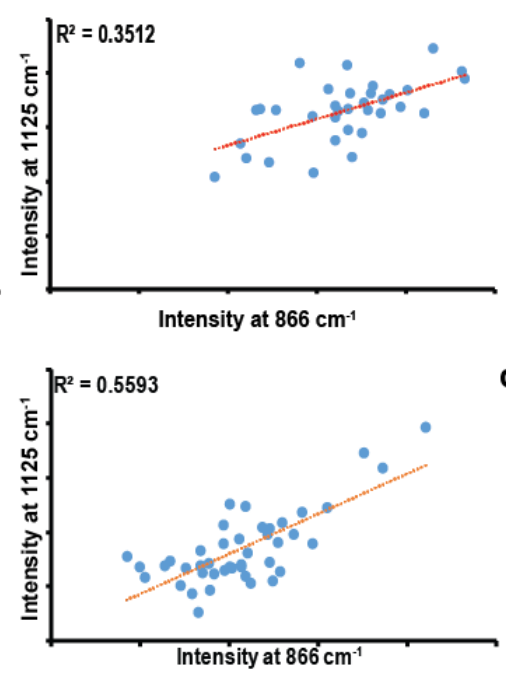

NMCSC
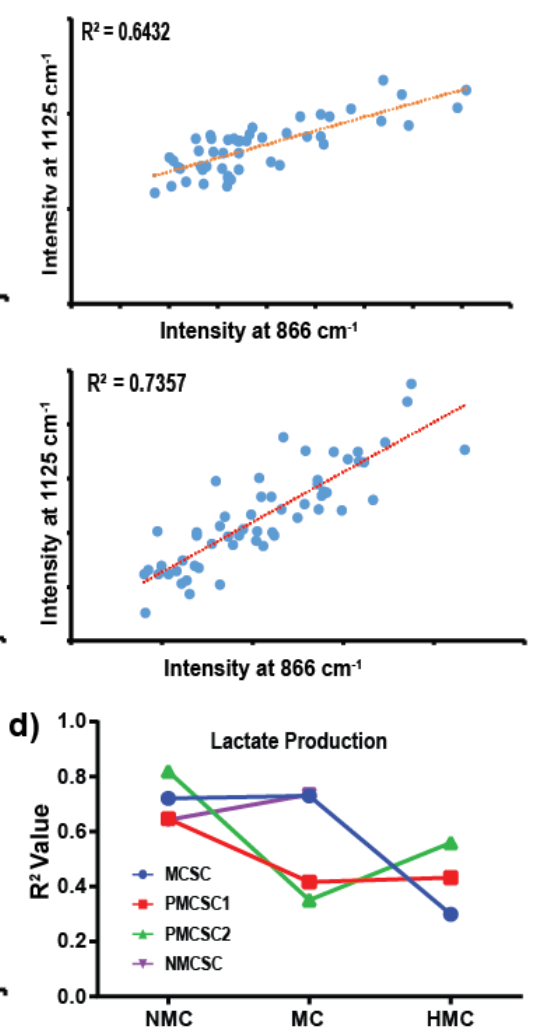

Figure S7: Single-cell Regression analysis of lactate-metabolite signals from different cancers progeny and non-metastatic stem cell population. 


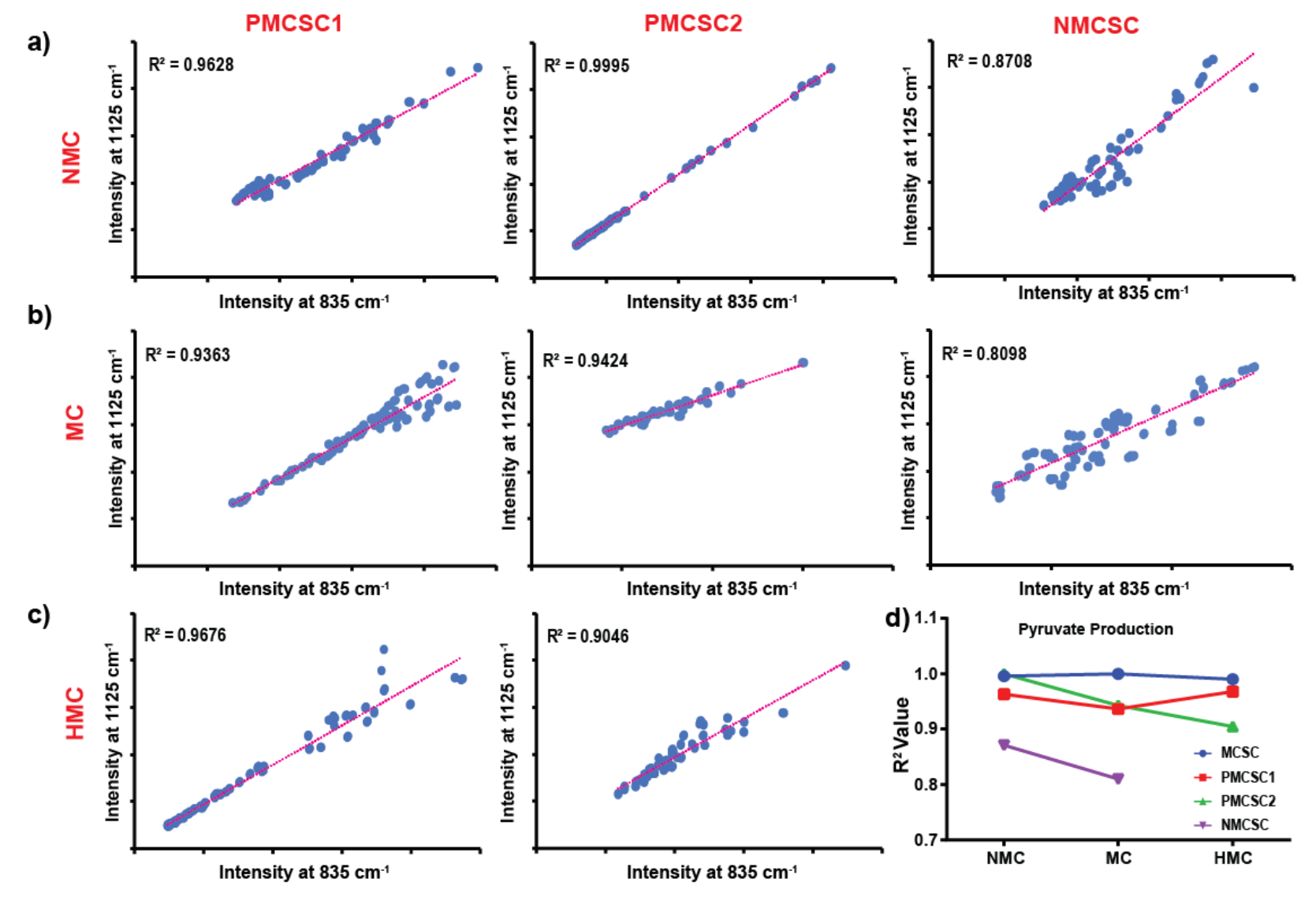

Figure S8: Single-cell Regression analysis of pyruvate-metabolite signals from different cancers progeny and non-metastatic stem cell population. 


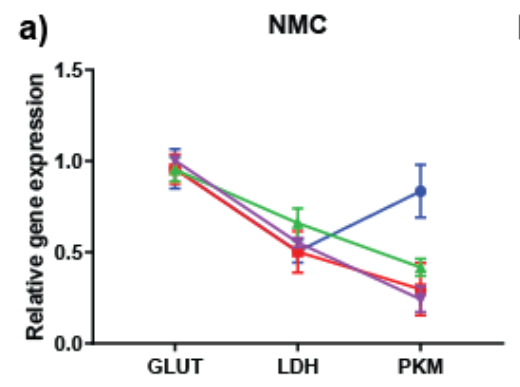

b)

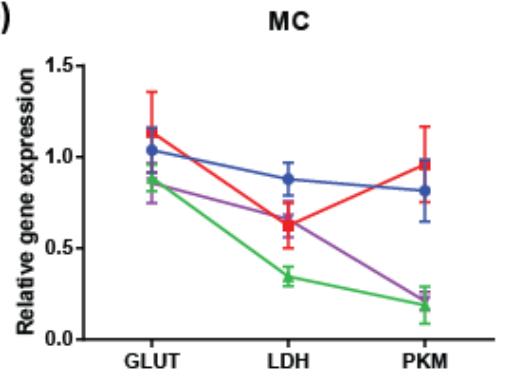

d)

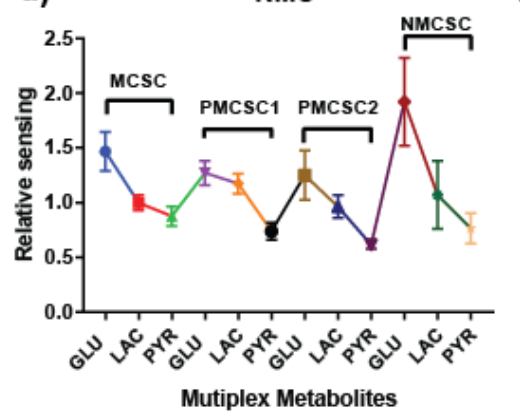

e)

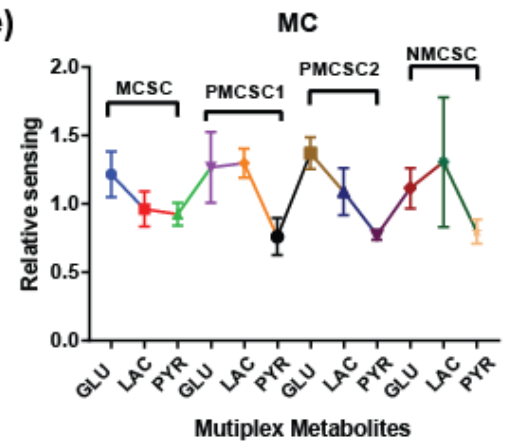

c)

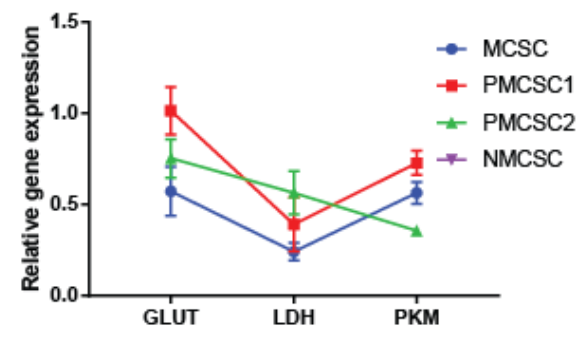

f)

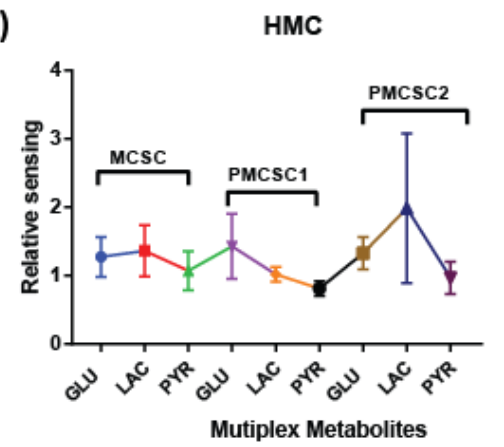

Figure S9: Metabolite adaptation studied using (a-c) relative gene expression and d-f) Raman analysis of multiple metabolites (glucose, lactate, and pyruvate) from MCSC, progeny, and NMCSC from different metastatic cancer phenotypes.

a)

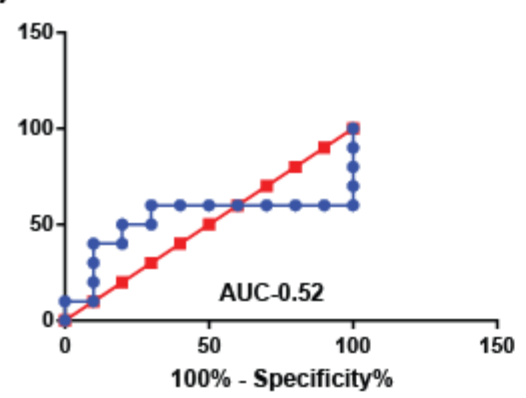

b)

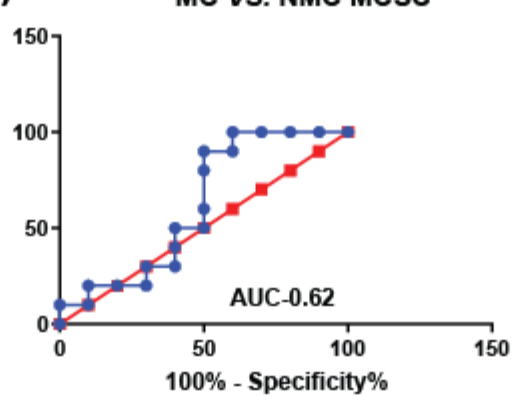

c) HMC vs. MC MCsC

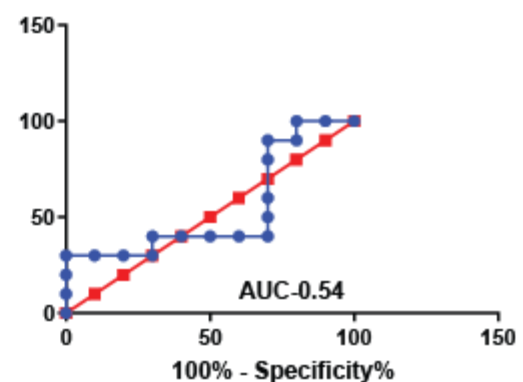

Figure S10: Cumulative ROC analysis further validates and confirms reduced distinctiveness and analogous features in MCSCs, facilitating the prediction of metastasis. 